\title{
Procesos de preparación para tocar en vivo: relatos de
}

\section{guitarristas de excelencia}

\author{
Rafael Iravedra, Daniel Wolff
}

\author{
Universidade Federal do Rio Grande do Sul | Brasil
}

Resumo: A pesar de que el proceso de aprendizaje de una obra musical tiene consecuencias directas en los resultados que un intérprete tendrá sobre el escenario, podemos diferenciar el proceso de aprendizaje de una obra del proceso de preparación para tocarla en vivo. Este trabajo presenta el análisis de 23 entrevistas y cuestionarios con guitarristas de excelencia sobre aspectos referentes a la preparación para tocar en púbico. El análisis siguió las directivas propuestas por Miles y Huberman (1994), partiendo de la interacción constante entre las fases de reducción de la información, disposición de los datos y verificación de conclusiones. Los resultados mostraron la importancia de la práctica de la performance, realizada a través de simulaciones, recitales informales y grabación de la propia ejecución. Otros aspectos que se mostraron relevantes fueron la creación de una imagen artística de la obra, la digitación, la visualización, la memorización y las rutinas pre-recital.

Palabras clave: Preparación de la performance. Performance en vivo. Performance de excelencia. Guitarra clásica.

\begin{abstract}
Even though the process of learning a musical work is inseparable from the results that a performer will achieve on stage, it is possible to distinguish the process of learning a piece from that of preparing it for a live performance. This study presents the analysis of 23 interviews and questionnaires conducted with acclaimed classical guitarists about strategies for preparing for public performances. We applied three processes of qualitative analysis as developed by Miles and Huberman (1994), namely data reduction, data display and conclusion drawing. The results showed the importance of practicing performances (through performance simulation, informal performances and self-recording). Other important aspects regarding this topic were the creation of an artistic image of the piece, fingerings, visualization, memorization and pre-concert preparation.
\end{abstract}

Keywords: Performance Planning. Live Performance. Expertise in Performance. Classical Guitar. 


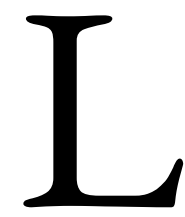

a performance musical en vivo es un momento de comunicación directa entre los intérpretes y el público, resultante de un largo proceso de estudio y preparación. Para Doğantan-Dack (2012, p. 36)1, “el músico clásico depende de la performance en vivo para establecer y definir su identidad artística como músico”. Dentro de las habilidades necesarias que los intérpretes deben dominar, podemos mencionar el control de la ansiedad escénica, el dominio técnico e interpretativo de las obras, y la capacidad de mantener el foco absoluto en la ejecución, evitando todo tipo de pensamientos dispersos. Chaffin y Logan (2006) comentan la necesidad de mantener la interpretación flexible y comunicativa emocionalmente con el público, al mismo tiempo que debe ser practicada de la forma más detallada posible para automatizarla. Los factores que pueden producir experiencias negativas sobre el escenario pueden ser varios: lapsos de memoria, sucesión de errores que generan un grado mayor de ansiedad, temblor y sudoración excesiva, respiración entrecortada, incremento de la tensión muscular y falta de concentración, generalmente productos de una preparación deficiente.

A pesar de que el proceso de aprendizaje de una obra musical tiene consecuencias directas en los resultados que un intérprete tendrá sobre el escenario, podemos diferenciar el proceso de aprendizaje de una obra - o sea, la resolución de las dificultades técnicas ${ }^{2}$ y la creación de una idea interpretativa- del proceso de preparación de una obra para tocarla en vivo. Según Doğantan-Dack (2012, p. 36), “existen importantes diferencias fenomenológicas, estéticas y, de hecho, existenciales entre la experiencia de una performance en vivo y en un estudio de grabación”. Durante el proceso de formación de un intérprete como performer, suele ser usual que muchas de estas diferencias no sean consideradas, colocando toda la atención en el trabajo técnico-interpretativo de la obra, dejando de lado aspectos importantes relacionados con la performance en vivo, como el desarrollo de las ideas expresivas considerando la comunicación con el público, las características acústicas de las diferentes salas, y otros elementos como actitud escénica (stage bebaviour), lapsos de memoria o ansiedad (JØRGENSEN, 2004, p. 95). Por el contrario, los músicos de excelencia utilizan diversos métodos, además de aquellos relacionados con el desarrollo de las habilidades motoras y el aprendizaje de

\footnotetext{
${ }^{1}$ Todas las traducciones son nuestras.

${ }^{2}$ Utilizaremos en este trabajo el concepto de técnica propuesto por Eduardo Fernández (2001, p. 14). El autor define la técnica como "la capacidad concreta de poder tocar un pasaje determinado de la manera deseada". Esta capacidad implica el dominio de los diferentes mecanismos necesarios en la ejecución instrumental para la realización de una idea interpretativa.
} 
repertorio específico. (PARTINGTON, 1995; CLARK; LISBOA; WILLIAMON, 2014, p. 20).

La preparación de músicos profesionales para la performance, dentro de la música clásica, ha sido abordada a partir del análisis de la organización del proceso de preparación de una obra y las características de las diferentes etapas (HALLAM, 1995a, 1995b, 2001; CHAFFIN et al., 2003; CLARKE et al., 2005; VINEY; BLOM, 2015; LISBOA; DEMOS; CHAFFIN, 2018); la importancia de la memorización para la ejecución y estrategias utilizadas por los músicos para memorizar (CHAFFIN; IMREH, 1997, 2002; HALLAM, 1997; CHAFFIN; LOGAN, 2006; CHAFFIN, 2007; CHAFFIN et al., 2010; GINSBORG; CHAFFIN, 2011; GINSBORG; CHAFFIN; DEMOS, 2012); la utilización de grabaciones como referencia (LISBOA et al., 2005; VOLIOTI; WILLIAMON, 2017); los aspectos expresivos en la construcción de la interpretación (VAN ZIJL; SLOBODA, 2011; DEMOS et al., 2018); y los procesos cognitivos en la construcción de la idea interpretativa (BANGERT et al., 2014; HÉROUX, 2016, 2018).

Dentro de la bibliografía guitarrística ${ }^{3}$, los aspectos propios de la preparación para tocar en vivo no siempre son considerados. Generalmente, estos métodos y tratados colocan el énfasis en los aspectos técnicos y mecánicos del instrumento. De todas formas, en los últimos años el tema es abordado con diferentes grados de profundidad (SHERROD, 1980; RYAN, 1991; SHEARER, 1991; PROVOST, 1992; TENNANT, 1995; GLISE, 1997; ISBIN, 1999; IZNAOLA, 2001; AZABAGIC, 2003; MASTERS, 2010; PEDREIRA, 2011; VILOTEAU, 2012; KÄPPEL, 2016; LEISNER, 2018). Teniendo en cuenta las temáticas relacionadas a la preparación para la performance en vivo, podemos agrupar la información tratada en dicha bibliografía en seis tópicos: (a) las características de las diferentes etapas en la preparación de una obra para la performance; (b) la práctica de la performance; (c) la visualización y la preparación mental para la performance; (d) la importancia de la memorización para tocar en público y estrategias para trabajarla; (e) estrategias para lidiar con el miedo escénico; $\mathrm{y}(\mathrm{f})$ rutinas previas a la performance.

Consideramos que estos tópicos podrían ser profundizados a partir del análisis sistematizado de relatos de experiencias de guitarristas de excelencia. Varios aspectos cruciales en la formación de un intérprete, como por ejemplo las rutinas pre-performance, generalmente son mencionados en espacios

\footnotetext{
${ }^{3}$ Llamaremos bibliografía guitarrística a los métodos y tratados de guitarra clásica, escritos por concertistas y pedagogos reconocidos. Usualmente estos textos son escritos con fines didácticos, y se corresponden a la literatura específica de guitarra utilizada por estudiantes durante su formación.
} 
como masterclass o charlas informales, pero no suelen ser analizados en profundidad en la literatura.

\section{Materiales y métodos}

Para indagar sobre el tema, buscamos obtener relatos de guitarristas considerados referencia internacional y con una consolidada carrera como recitalistas. En la opinión de Woody (2004, p. 18), estudiar a los músicos de excelencia es estudiar lo que funciona, ya que son la prueba de lo que es necesario para alcanzar un alto nivel en la interpretación. Consideramos que todos los guitarristas entrevistados en este estudio, por los motivos anteriormente mencionados, se encuadran dentro de la definición dada por Papageorgi (2014, p. 303). La autora postula que podemos conceptualizar a este tipo de músicos como:

una persona que consistentemente demuestra niveles de performance excepcionales comparado con otros individuos de edad o experiencia similar, y cuyo nivel de habilidad puede ser confirmado por algún tipo de resultado mensurable (como los resultados de un examen o audición, o el reconocimiento por parte de otros especialistas y/o del público).

En la primera etapa de este trabajo fue realizada una entrevista semiestructurada con la concertista María Isabel Siewers abordando diferentes temáticas relacionadas al proceso de preparación para tocar en vivo. El guión fue realizado a partir de categorías que abarcaban aspectos generales y amplios, ya que la investigación se encontraba en una fase exploratoria y nos interesaba conversar sobre el proceso de preparación de Siewers en general. El análisis de esta entrevista fue clave para poder establecer algunas categorías, y nos permitió pensar cuales aspectos serían más significativos de abordar con el resto de los concertistas.

Para alcanzar la máxima cantidad de participantes posible, enviamos por correo electrónico cuestionarios con diez preguntas abiertas relacionadas a las principales estrategias utilizadas en la preparación para tocar en vivo, la importancia de la memorización, el estudio mental, las rutinas previas a tocar en público, así como experiencias positivas y negativas sobre el escenario. Para facilitar las respuestas, ofrecimos la posibilidad de responder por escrito, por mensaje de audio, en comunicación vía Skype o similar, de acuerdo a las preferencias de cada uno de los guitarristas. Los cuestionarios fueron enviados en portugués, español e inglés, según la nacionalidad de cada 
entrevistado. De los 15 participantes que respondieron, 14 lo hicieron de forma escrita y solo uno envió sus respuestas en audio (Tabla 1).

TABLA 1 - Cuestionarios: participante, fecha y formato de respuesta

\begin{tabular}{ccc}
\hline Participante & Fecha de envío & Formato de respuesta \\
\hline Alice Artzt & 10 de abril de 2017 & escrito \\
Eduardo Fernández & 10 de abril de 2017 & escrito \\
Edson Lopes & 11 de abril de 2017 & audio \\
Stefano Grondona & 12 de abril de 2017 & escrito \\
Stanley Yates & 12 de abril de 2017 & escrito \\
Scott Borg & 18 de abril de 2017 & escrito \\
Frank Bungarten & 23 de abril de 2017 & escrito \\
Thomas Viloteau & 26 de abril de 2017 & escrito \\
Carlos Pérez & 5 de mayo de 2017 & escrito \\
Lucio Matarazzo & 9 de mayo de 2017 & escrito \\
Jason Vieaux & 26 de mayo de 2017 & escrito \\
Carlo Marchione & 29 de junio de 2017 & escrito \\
Sérgio Assad & 1 de septiembre de 2018 & escrito \\
Jonathan Leathwood & 2 de septiembre de 2018 & escrito \\
Denis Azabagic & 11 de septiembre de 2018 & escrito \\
\hline
\end{tabular}

Fuente: Los autores.

Luego del análisis de los primeros cuestionarios recibidos, observamos la necesidad de profundizar algunos de los conceptos obtenidos en las respuestas, especialmente los relacionados con las rutinas pre-performance, así como las estrategias específicas que estos guitarristas utilizan para prepararse para tocar frente al público. De esta manera, realizamos nuevas entrevistas semiestructuradas de forma presencial a otros guitarristas, lo que permitió la posibilidad de repreguntar y profundizar en las ideas que más nos interesaban. El contacto fue hecho por e-mail, y todos los músicos consultados accedieron a participar de la investigación. Fueron realizadas siete entrevistas semiestructuradas más (Tabla 2), cuyo guión fue elaborado a partir de los cuestionarios enviados anteriormente a los otros participantes. Todas las entrevistas fueron transcriptas, editadas y revisadas para su posterior análisis. Las transcripciones fueron enviadas para los participantes junto con una carta de consentimiento informado para la utilización de los datos en esta investigación. 
TABLA 2 - Entrevistado, fecha, lugar y duración de las entrevistas

\begin{tabular}{cccc}
\hline Entrevistado & Fecha & Lugar & Duración \\
\hline María Isabel Siewers & 27 de enero de 2017 & Pelotas/RS, Brasil & $60^{\prime}$ \\
Zoran Dukic & 30 de octubre de 2017 & Aveiro, Portugal & $20^{\prime}$ \\
Margarita Escarpa & 8 de noviembre de 2017 & Vigo, España & $40^{\prime}$ \\
Àlex Garrobé & 28 de noviembre de 2017 & Barcelona, España & $58^{\prime}$ \\
José Antonio Escobar & 28 de noviembre de 2017 & Barcelona, España & $52^{\prime}$ \\
Dejan Ivanović & 28 de diciembre de 2017 & Guimarães, Portugal & $64^{\prime}$ \\
Fabio Zanon & 9 de febrero de 2018 & Entrevista en vivo, vía Skype & $39^{\prime}$ \\
Marco Tamayo & 10 de marzo de 2018 & Salzburgo, Austria & $52^{\prime}$ \\
\hline
\end{tabular}

Fuente: Los autores.

De esta manera, obtuvimos 23 relatos en total (entre cuestionarios abiertos y entrevistas en vivo) para la presente investigación. Como señala Ruquoy (1997, p. 103), cuando hablamos de investigaciones cualitativas, el número de entrevistados no constituye un factor relevante. Los participantes no son elegidos por la relevancia numérica, sino por su carácter paradigmático. En el caso de este trabajo, todos los músicos son considerados concertistas de excelencia, y cada relato es valioso y relevante. Por ese motivo, los participantes no fueron considerados sujetos anónimos por los autores y cada cita utilizada está identificada.

\section{Procedimiento de análisis de los datos}

Como señala Pereira Coutinho (2011, p. 216), es usual que los estudios cualitativos produzcan una enorme cantidad de información descriptiva debido a su carácter abierto y flexible. Esta información necesita ser organizada y "reducida" (data reduction) para posibilitar la descripción e interpretación del fenómeno estudiado, y de esa forma "dar sentido" a la enorme cantidad de datos. Para el análisis de las entrevistas realizadas en nuestra investigación, seguimos las directivas propuestas por Miles y Huberman (1994). De acuerdo con estos autores, el proceso de análisis puede ser entendido como la interacción constante entre las fases de reducción de la información, disposición de los datos y extracción o verificación de conclusiones. La reducción de la información implica elegir, focalizar, abstraer, simplificar y/o transformar los datos que aparecen en el gran corpus de material empírico a ser analizado, "la reducción de la información no es un elemento separado del análisis, es parte del análisis" (MILES; HUBERMAN, 1994, p. 11). Posteriormente, toda la información se 
organiza y sintetiza en gráficos y matrices descriptivas y explicativas que permiten avanzar en el proceso de análisis y en la elaboración de conclusiones. Finalmente, en la última fase (drawing conclusions), se identifican regularidades, patrones, explicaciones y proposiciones (MILES; HUBERMAN; SALDAÑA, 2014, p. 10), de la misma forma que se elaboran generalizaciones, tipologías y modelos (SABARIEGO; VILÀ; SANDÍN, 2014, p. 122). Según Mejía Navarrete (2011, p. 57), en esta fase es necesaria la interpretación a partir de enunciados de carácter conceptual y explicativo de los datos cualitativos.

Como herramienta auxiliar, utilizamos el programa de análisis cualitativo ATLAS.ti, lo que facilitó enormemente el manejo de la información a partir de la creación de categorías, códigos, anotaciones y reflexiones; agrupamiento de códigos en familias; búsqueda y recuperación de datos; y finalmente, la creación de reportes para cada uno de los códigos establecidos. ATLAS.ti es un software de análisis cualitativo que pertenece a los programas del tipo CAQDAS (Computer Assisted Qualitative Data Analysis Software). Dabenigno (2017, p. 51) señala dos ventajas en la utilización de este tipo de programas: en primer lugar, la posibilidad de contar con un archivo integral que contiene todos los documentos a analizar; y en segundo lugar, la versatilidad que permiten para ir generando el listado y la reducción del tiempo de codificación, si comparamos con procedimientos manuales.

\section{Resultados y discusión}

Luego del análisis de los relatos de los guitarristas entrevistados, agrupamos la información en diferentes tópicos a partir de los aspectos que consideramos más relevantes durante la preparación para tocar en vivo: la preparación técnica y artística, la visualización y el estudio mental, la importancia de la memorización al tocar en vivo, la práctica de la performance, la preparación próxima a la performance, y rutinas en la sala de conciertos.

\subsection{Algunas consideraciones sobre la preparación técnica y artística}

Varios de los entrevistados comentaron la importancia de realizar un abordaje de la obra desde el punto de vista musical, ya desde el primer contacto con la partitura, a partir de una "comprensión 
de todos los aspectos relacionados con esa composición” (ASSAD, 2018, comunicación personal). Para Matarazzo (2017, comunicación personal), es indispensable desde el comienzo de la preparación "conocer muy bien la forma musical, la estructura y las frases y elementos de la cual [la obra] está formada”. De la misma forma, Fernández (2017, comunicación personal) considera que "debe tenerse una imagen clara de lo que se quiere conseguir en términos de sonido, incluso antes de empezar a encarar el trabajo instrumental". Podemos observar una relación próxima con la idea de "imagen artística” o big picture propuesta por Neuhaus en su libro The Art of Piano Playing (1958/1973). Según Neuhaus (1973), la imagen artística puede definirse como la representación mental que el intérprete forma de la obra. O dicho en otras palabras, cómo la obra debería sonar. Esta es una idea similar a cómo los especialistas de otras áreas se aproximan a un nuevo problema, considerándolo en su totalidad, a partir de la big picture (CHAFFIN; LOGAN, 2006, p. 116). De esta manera, se busca una solución - a pesar de todavía desconocida-, ya que los especialistas comprenden los aspectos implicados en la problemática, a diferencia de los principiantes que usualmente se pierden en los detalles (LISBOA; DEMOS; CHAFFIN, 2018, p. 552).

La creación de una imagen artística de la obra, influye tanto en las decisiones interpretativas como en las digitaciones y en la selección y resolución de los pasajes más problemáticos de la obra. Garrobé (2017) destaca la importancia de identificar, desde un primer momento, los puntos más difíciles de resolver, sean mecánicos, formales o expresivos. De esta forma, el trabajo no comienza en la primera nota y termina en la última, sino que establece una jerarquía de estudio comenzando por las principales dificultades, de tal forma que pueda absorber la pieza cuanto antes. Para Ivanović (2017), en la primera etapa de trabajo (que llama "base de construcción de la pieza"), es necesario realizar un análisis estructural de la obra y, a partir de esto - y también considerando otros aspectos como carácter musical y estilo-, definir una digitación según la idea musical y el tempo final de la pieza.

De esta forma, observamos que en la guitarra, la digitación de una obra tiene una importancia fundamental dentro del proceso de preparación. Es necesario que la digitación responda siempre a la idea interpretativa y a las características ergonómicas de cada intérprete. Borg (2017, comunicación personal), en relación a este tema, comenta: "no suelo tomar al pie de la letra las digitaciones marcadas en la partitura y pruebo una gran cantidad de opciones hasta dar con la más adecuada”. Como señala 
Fernández (2017, comunicación personal):

\begin{abstract}
$\mathrm{Al}$ tomar el instrumento, uno tiene que saber qué es lo que se quiere conseguir; esto puede referirse a un pasaje, una frase, una sección de la obra. De esta manera, es posible llegar a establecer una digitación que sea compatible con el objetivo musical, y trabajar con el instrumento hasta conseguir lo que se desea. Este proceso no es necesariamente lineal, hay idas y venidas, refinamientos y modificaciones de la idea musical, ajustes de la digitación para responder a ellos. Por supuesto, establecer este objetivo implica al menos un cierto conocimiento del estilo, al menos algo de análisis, y otros aspectos puramente musicales, a los cuales lo técnico debe estar estrictamente subordinado.
\end{abstract}

Es importante destacar también que muchas veces la "digitación ideal” puede no ser la mejor digitación para tocar la pieza en público. Varios de los entrevistados comentaron la importancia de elegir una digitación que funcione sobre el escenario, incluso cuando estemos un poco fríos o nerviosos.

Yo digito distinto, o redigito si he trabajado una obra primero para tocarla en público sin idea de grabarla. Redigito, quiero decir busco digitaciones más perfectas, aunque sean un poco más difícil [...] O que, aunque la digitación está muy bien, es un pasaje no tan fácil. Pero bueno, aun así, piensas que lo vas a poder hacer y luego te resulta que, claro entre que tienes los focos delante, entre que vas con la ropa de concierto, que normalmente no usas los tacones cuándo estás en casa y tal, pues son demasiadas sorpresas. Entonces dices 'no, aquí hay que facilitar'. (ESCARPA, 2017, información verbal)

Un aspecto fundamental en la preparación es que los problemas técnicos de la obra deben estar resueltos para alcanzar un alto nivel interpretativo sobre el escenario. Para Matarazzo (2017, comunicación personal) "solo si tienes un dominio técnico total de los movimientos de las manos y de la alternancia entre la tensión y la relajación de esos movimientos (y del cuerpo entero) en el escenario, puedes pensar en la música que vas a tocar”. Igualmente, Azabagic (2018, comunicación personal) afirma que "si en la obra que tocamos hay problemas técnicos que no están resueltos, entonces no hay una base en donde construir cualquier paso posterior”. La adquisición del dominio técnico de la pieza permite que el intérprete solo se preocupe en buscar "su estado emocional" (GARROBÉ, 2017, información verbal). En esa búsqueda de la perfección técnica, Artzt (2017) comenta que una estrategia que utilizaba ${ }^{4}$ durante la preparación, era imaginarse que tocaba sobre una trampa con cocodrilos debajo: "practicaba de forma lenta, sonora y con mucho cuidado, porque

\footnotetext{
${ }^{4}$ Alice Artzt se encuentra retirada de la actividad concertística desde hace más de veinte años.
} 
si cometía algún error, caería en el foso iy los cocodrilos me iban a comer!” (ARTZT, 2017, comunicación personal). Tamayo (2018, información verbal) relata una estrategia similar destacando la importancia de que cada repetición sea única y perfecta: "yo estudio visualizando, por ejemplo, que estoy sentado ahí en el medio del escenario, o en el medio de la grabación, yo estudio que ahí tengo a Beethoven, o que tengo un manager que me promete no sé qué y que tengo que tocar bien, esas presiones”. Este dominio técnico también permite alcanzar altos niveles de concentración, necesarios para un buen desempeño sobre el escenario, y para tocar "solo pensando en la expresividad musical" (MATARAZZO, 2017, comunicación personal).

Marchione (2017, comunicación personal) destaca la importancia de considerar las capacidades instrumentales de cada intérprete a la hora de elegir el repertorio, no solo por el nivel de dificultad sino por las características de cada lenguaje, "de la misma forma que muchos cantores aman a Wagner, pero no tienen una inclinación natural para cantarlo y, en que caso de hacerlo podrían arruinar su voz”. Otro aspecto relevante es la conexión que cada músico tiene con la obra. Assad (2018, comunicación personal) menciona que "antes de elegir una pieza para la performance, tenemos que tener un amor verdadero por esa obra en particular”. De la misma forma, para Leathwood (2018, comunicación personal), uno de los aspectos más importantes en la preparación del repertorio es cultivar "una conexión profunda y perdurable con la obra”. Por su parte, Garrobé (2017) plantea la necesidad de que la pieza - que es externa a nosotros - tenga un sentido para el intérprete, que le permita absorber su contenido y hacerlo propio. Esta afinidad también influirá en la cantidad de tiempo necesario para preparar una obra.

La utilización de otras grabaciones durante la preparación de una obra es un tema polémico dentro de la tradición de la música clásica. Para algunos autores, constituye una herramienta de gran valor en la formación de un intérprete. Por ejemplo, Freitas y Gerling (2016, p. 86) expresan que "los modelos aurales en forma de grabaciones también incrementan las dimensiones expresivas en la performance". Las autoras, en un estudio con dos estudiantes preparando Ponteio 46 del compositor Camargo Guarnieri, afirman que el uso de las grabaciones de intérpretes considerados referencia, posibilitó que los participantes pudieran construir estrategias de estudio relacionadas a las figuraciones polirrítmicas, ampliar el vocabulario de recursos expresivos y definir de forma más consciente el carácter de la obra elegida. Por el contrario, Volioti y Williamon (2017, p. 500) afirman 
que en muchos casos "las grabaciones han sido consideradas negativamente por supuestamente sofocar la originalidad artística y reducir significativamente la individualidad y variabilidad de la interpretación”. Con respecto a este tema, algunos de los entrevistados comentaron utilizarlas para escuchar lo que hacen otros intérpretes e incorporar algunos recursos a la propia performance, o para pensar alternativas e incluso, descartar más rápidamente ideas que no funcionan. Garrobé (2017) recomienda a sus alumnos la utilización de grabaciones, pero solo luego de haber construido por lo menos una primera idea interpretativa. "Lo ideal es que tú te haces tu versión, luego escuchas versiones de maestros y dices 'ah, me interesa por aquí, me interesa por allá. En todo caso corriges lo que has hecho o ves mejores opciones” (GARROBÉ, 2017, información verbal). Para Escobar (2017, información verbal), el uso de grabaciones es una herramienta muy útil, especialmente cuando el tiempo disponible para la preparación de una obra es escaso: "Si tengo que trabajar rápido también escucho más grabaciones. No trato de imitar exactamente, pero sí tener una idea, [...] Y ahí es buena la tecnología, cosas que antes uno tenía que resolver en la pura intuición, ahora está YouTube y otras plataformas.”

Por su parte, Azabagic (2018, comunicación personal) también destaca la importancia de la utilización de grabaciones como modelo y las posibilidades que ofrecen para la construcción de una interpretación:

\footnotetext{
Para mí es importante sentir el estilo y aprender de otros músicos. Hay tantos grandes músicos de los que he aprendido: compañeros de concursos, profesores y muchos músicos que escuché tocar, escuché sus grabaciones... Aprendiendo de los mejores, escuchando lo que están haciendo, escuchando las ideas que se les ocurren (técnica y musicalmente), e incorporándolas a mi propia interpretación.
}

\subsection{Sin el instrumento: la visualización y el estudio mental}

La utilización de la visualización y el estudio mental aparece en los relatos a lo largo de todas las etapas de preparación de una obra y con varias finalidades diferentes. Cabe destacar que estos dos términos, a pesar de reflejar ideas similares, pueden entenderse con algunas diferencias. En este trabajo tomaremos el sentido expuesto por García en su libro “Entrenamiento mental para músicos” (2017). Para este autor: 
Cuando nos referimos al estudio mental dentro del ámbito musical, hablamos por lo general de aspectos como leer mentalmente las notas de una partitura, analizar o memorizar. [...] La visualización tiene que ver con el acto de representarse algo, sean elementos perceptivos (imágenes visuales, sonoras, cenestésicas...) o una situación (los movimientos que se realizan al interpretar, un concierto en público...). Por lo tanto, el estudio mental incluye la visualización, y es el término que se suele utilizar en la música para referirnos a este tipo de práctica. (GARCÍA, 2017, p. 17)

La mayor parte de los entrevistados destaca la importancia del estudio mental — "uno debe tener un total entendimiento de sus movimientos para imaginarse tocando sin el instrumento. Esto es crucial” (BUNGARTEN, 2017, comunicación personal); “es absolutamente crucial usar el estudio mental” (LEATHWOOD, 2018, comunicación personal)—, incluso aquellos que no lo utilizan o lo utilizan muy poco.

Dentro de los objetivos mencionados en la utilización de esta estrategia se encuentran: aprendizaje de una obra nueva (especialmente en viajes o períodos con poco tiempo para estudiar); como herramienta de memorización; para resolver problemas técnico-interpretativos; y como fuente de nuevas ideas interpretativas. Yates (2017) comenta la utilización del estudio mental como preparación para la parte física, especialmente en obras muy difíciles. Considerando los procesos de memorización, la visualización aparece como un recurso fundamental y que posibilita sentir una gran seguridad sobre el escenario. El estudio mental permite también resolver problemas técnicos o interpretativos. Pérez (2017, comunicación personal) comenta que en algunas ocasiones visualiza algunos pasajes o intenta solucionar alguna traba mecánica a partir del estudio mental, "en especial cuando hay que reaprender una sección, corregirla o modificarla en el plano técnico o en las ideas musicales". Los guitarristas entrevistados comentan que una de las principales ventajas de este tipo de estudio es que, al no estar presentes las limitaciones físicas del instrumento, es más fácil tomar conciencia de todos los parámetros envueltos en la ejecución. Esa falta de limitaciones fúsicas permite imaginar nuevas ideas interpretativas, ya que "la práctica con el instrumento puede hacer que nuestra imaginación se vuelva perezosa” (GRONDONA, 2017, comunicación personal).

El estudio mental es un recurso fundamental especialmente en el proceso de memorización de una obra, "la visualización es tan poderosa que, desde luego para memorizar, a mí me da una seguridad cien por cien” (ESCARPA, 2017, información verbal). Dukic (2017, información verbal) revela: “¿cómo averiguo si memorizo? me siento con la partitura en la mano, cierro los ojos y voy 
tocándola. Y cuando me pierdo, miro en la partitura [...] Es trabajo, es estudiar”. De la misma forma Azabagic (2017, comunicación personal) destaca:

\begin{abstract}
Mi memoria suele ser bastante buena, sin embargo, la memoria puede jugarte una mala pasada, especialmente si estás bajo presión. Un estudio mental te ayuda a asegurarte de no olvidar nada, incluso bajo mucha presión. Un estudio mental significa tocar la pieza en tu cabeza sin el instrumento asegurándote de conocer cada dedo que estás usando en ambas manos. Si no puedes recordar un pasaje específico, debes tomar la partitura, verificarlo y reiniciar el estudio hasta que ese pasaje esté completamente asimilado.
\end{abstract}

\title{
3.3 La importancia de la memorización al tocar en vivo
}

Dentro de las investigaciones empíricas, varios autores destacan la importancia y las ventajas que tiene la memorización en la ejecución musical en vivo (CHAFFIN; IMREH, 1997; CHAFFIN et al., 2010; HALLAM, 1997). Sin embargo, en el caso de los guitarristas entrevistados, podemos observar que las opiniones sobre el tema no son unánimes. Varios de los entrevistados comentaron que la consideran un aspecto muy relevante que favorece el lenguaje corporal (MARCHIONE, 2017), los aspectos comunicacionales (IVANOVIĆ, 2017; YATES, 2017), la espontaneidad (IVANOVIĆ, 2017), y la posibilidad de tener todos los sentidos enfocados en la música (ESCARPA, 2017). Para Borg (2017, comunicación personal), las performances donde el intérprete utiliza la partitura generalmente son "un poco planas y con un énfasis mayor en las notas que en la narrativa de la partitura", y considera "vital" la memorización al tocar en vivo. Ya otros entrevistados, a pesar de considerarla importante, no la entienden como obligatoria, especialmente cuando se trata de música de cámara o con orquesta.

Creo que en los conciertos de música de cámara y en los conciertos con orquesta, terminamos gastando una energía muy grande para memorizar detalles cuando, en fin, uno está tocando acordes, arpegios, solo de relleno y terminamos descuidando el tocar la obra bien. Entonces creo que cuando uno toca un concierto de, digamos, Castelnuovo-Tedesco, hay algunos rellenos que uno puede leer, y los pasajes realmente de bravura, pasajes solistas, naturalmente uno los memoriza porque los estudia mucho. (ZANON, 2018, información verbal)

Varios de los guitarristas consideran que la memorización no es un aspecto determinante y que "es perfectamente posible tocar con la partitura y hacer una performance espontánea” 
(FERNÁNDEZ, 2017, comunicación personal) y "que no hay que tocar de memoria, podemos tocar perfectamente bien con la partitura” (DUKIC, 2017, información verbal). Lopes (2017), Yates (2017) y Escobar (2017) comentan que muchas veces prefieren leer, ya que el miedo de que ocurra algún lapso de memoria puede interferir en la ejecución. Lopes, por ejemplo, utiliza un tablet como herramienta de lectura sobre el escenario 5 . Escobar (2017) también destaca la ventaja de poder abordar una mayor cantidad de repertorio al tocar con la partitura.

Mira, yo ya hace tiempo que tomé la determinación de que prefiero tocar mucha más música, aunque no sea de memoria. Pero la vida es muy corta y no quiero estar un año, dos años, tocando lo mismo. Entonces yo en un año toco, no sé, treinta programas o más. Leo mucha música también porque me gusta leer la música, así como leer libros. Creo que es algo importante, que hay que hacer. (ESCOBAR, 2017, información verbal)

\subsection{La práctica de la performance}

Dentro del proceso de preparación para tocar en vivo, la mayor parte de los entrevistados comentó la importancia de diferenciar el proceso de aprendizaje de una obra, del proceso de preparación para tocarla en público. Para Grondona (2017, comunicación personal), "el punto principal, o la 'estrategia' es prepararnos para seguir un itinerario, sabiendo que cada paso puede ser hecho como nunca antes”. Zanon (2018) también menciona la necesidad de seguir un itinerario (un "guión mental") que debe ser construido a lo largo del aprendizaje de una obra. Este itinerario permite al intérprete establecer puntos de referencia (lo que Chaffin denomina "guías de ejecución”) que sirven para poder lidiar con la espontaneidad que la ejecución musical en vivo necesita, a partir de la automatización de aspectos técnicos, interpretativos y expresivos, que deben ser trabajados durante todo el proceso de preparación.

Considerando las diferencias entre el estudio de una obra y el estudio de la performance de esa obra, Zanon (2018, información verbal) sostiene que el hecho de tocar bien una obra "no quiere decir

\footnotetext{
${ }^{5}$ Lo cual no excluye que puedan ocurrir accidentes al tocar en público, como por ejemplo problemas con el pedal utilizado para pasar las páginas de la partitura digital.

${ }^{6}$ Los guías de ejecución son puntos de referencia mentales de una obra que los intérpretes mantienen en la memoria durante la ejecución, en busca de un fluir musical continuo. Así, son elegidos y practicados por el intérprete y ofrecen una red de seguridad en caso de problemas de memoria. "Los guías de ejecución se vuelven una parte integral de la performance y proveen un medio de monitorear y controlar conscientemente las acciones rápidas y automáticas de las manos" (CHAFFIN; LOGAN, 2006, p. 115).
} 
que uno se sienta cómodo de tocar eso para un público [...], hay que hacer una simulación en algún momento”. Esa simulación es entendida y practicada con algunas diferencias por los entrevistados, pero la gran mayoría relató tocar el programa completo (con mayor o menor énfasis en recrear los aspectos de la performance en vivo) por lo menos los últimos días antes del concierto. Vieaux (2017, comunicación personal) recomienda "recrear la performance hasta el último detalle. Incluso dentro del cuarto, usar la puerta como entrada al escenario, practicar la caminata, sentarse, la afinación, todo". Zanon (2018, información verbal) en concordancia con esa idea, esclarece: “¡cómo sería esa simulación? Sería realmente el proceso de hacer todo el ritual que uno haría para tocar en público. O sea, colocar una silla como la que uno usaría para tocar en público, vestir la misma ropa, afinar, controlar el tiempo y la calidad de la afinación y hacer el precalentamiento.”

Garrobé (2017, información verbal) comenta la importancia de pensar en la totalidad del programa en términos discursivos — “como una argumentación”-, con un sentido global, y no como una sucesión de piezas sin relación, “eso multiplica el efecto de la pieza y también la comodidad con ellas”. Otro aspecto comentado por Garrobé (2017) y por Zanon (2018) es la cuestión física que debemos considerar al tocar en vivo, el cansancio que podemos sentir o las dificultades que puedan surgir a lo largo de la performance. En este sentido, Ivanović (2017) señala la importancia de considerar con detenimiento los puntos de tensión y relajación (musicales y físicos), no solo en cada obra, sino en el programa como un todo.

Dentro del proceso de la práctica de la performance, varios entrevistados también comentaron la importancia de grabarse. Para Zanon (2018, información verbal) “grabarse es la mejor manera, o una de las mejores, de tener una idea de lo que uno es capaz de hacer sobre el escenario”. Escobar (2017) plantea que esa herramienta le permite al intérprete analizar la ejecución desde un punto de vista externo y de esa forma, poder observar mejor los posibles problemas técnicos, interpretativos o físicos que se puedan tener.

¿Por qué uno puede ser tan buen crítico cuando escucha al otro? Porque justamente uno no está tocando, está viéndolo de afuera. Entonces una forma de salirte de ti, es grabándote. Tanto en audio primero, y por qué no en video para ver como están las manos. Hay que estar vigilando las manos, las mías especialmente [risas]. Yo no me considero una persona con muy buenas manos, entonces tengo que estar vigilando que no estoy levantando mucho los dedos. O de repente el hombro, alguna tensión. (ESCOBAR, 2017, información verbal) 
Otro aspecto importante dentro de la práctica de la performance son los conciertos informales: tocar para colegas, amigos o en espacios donde no haya una presión similar a una sala de conciertos. Leathwood (2018, comunicación personal) comenta que organiza conciertos "en lugares de 'bajo riesgo' uno o dos días antes de la performance”. Esos conciertos informales permiten tener una noción de los posibles problemas que pueden suceder en vivo. Artzt (2017) recomienda tocar enfrente de personas en diferentes situaciones y acostumbrarse a los problemas que pueden suceder, como sudor en las manos o lapsos de memoria. Por ejemplo, Viloteau (2017) relata que organiza su preparación en tres etapas: en primer lugar, el aprendizaje de la obra propiamente dicho; en segundo lugar, la realización de conciertos informales para identificar los posibles problemas que suceden bajo presión y la utilización de estrategias para solucionarlos; y en tercer lugar, la performance en vivo. Tanto Zanon (2018) como Escarpa (2017) relataron que crean dificultades extras (por ejemplo, tocar cansados o con una ropa más incómoda) durante la práctica con el objetivo de conocer el estado en que se encuentra cada obra y para acostumbrarse a lidiar con imprevistos.

Algunos de los concertistas afirmaron que no realizan ningún tipo de simulación de la performance. Matarazzo (2017) relata que apenas toca el programa completo el día anterior al concierto. Bungarten (2017, comunicación personal) manifiesta: "no, no hago [una simulación], ¿por qué debería? La performance es comunicación con la audiencia. Yo siento al público y me dejo llevar por la energía de los oyentes atentos”. Marchione (2017, comunicación personal) también reflexiona sobre este aspecto y comenta: "De hecho, prefiero hacer lo contrario, intento pensar en el escenario que estoy en mi casa [...]. Para mí, no funciona simular una perfomance en vivo si en casa no tengo lo más importante de eso: el público, la sala, el humor que tenga ese día...”

Cabe destacar que los relatos de estos tres guitarristas fueron a través de cuestionarios abiertos. De esa forma, no hubo posibilidad de repreguntar sobre ese aspecto. Es posible que, profundizando un poco más en una situación de entrevista semiestructurada, pudiésemos observar algún tipo de trabajo en relación a acostumbrarse a tocar el programa completo, a grabarse o tocar enfrente de personas en un ambiente más relajado. 


\subsection{La preparación próxima a la performance}

Partington (1995) identificó en sus entrevistas que durante los días previos a la perfomance los músicos utilizan estrategias musicales, físicas y mentales específicas para obtener los mejores resultados sobre el escenario. El autor enfatiza que esas rutinas son creadas a partir de las experiencias personales y, a pesar de necesarias, no constituyen atajos que substituyan a una buena preparación técnica y artística. Connolly y Williamon (2004) destacan que esas rutinas pre-performance son igualmente útiles para estudiantes de instrumento.

Varios de los entrevistados relataron que no tienen una rutina fija para el día del recital, y que esta suele adaptarse a las necesidades específicas en cada caso. Para Yates (2017, comunicación personal), "el hecho de estar de gira y viajando no permite la idea de una rutina el día de la performance. Generalmente me gusta estar solo en el hotel, tocar un poco, leer, ir a un café, etc.” De igual manera, Marchione (2017, comunicación personal) especifica: "mi rutina es no tener una rutina. Dejo mi sentimiento interior decidir qué hacer.” Otros concertistas relataron que no consideran el día del recital como un día especial, e intentan continuar con la rutina diaria: "intento actuar de la misma forma, no hago nada fuera de lo usual” (BORG, 2017, comunicación personal); “como lo mismo de siempre, todo el resto es lo mismo” (VIEAUX, 2017, comunicación personal); “el día del concierto debería ser un día normal, lo más normal posible, porque hay más días normales que los días de concierto” (TAMAYO, 2018, información verbal).

Observamos —en los guitarristas entrevistados para este estudio- la importancia, durante el día del concierto, del descanso, de la buena alimentación, y del uso de estrategias de pensamiento positivo y de motivación para prepararse de la mejor forma para enfrentar al público. Zanon comenta la necesidad de mantener una actitud mental favorable y evitar todo tipo de pensamientos intrusos que dificulten la concentración.

Tocar bien en público depende mucho de cumplir un cierto guión mental. Ese guión mental es algo que uno tiene que construir de a poco mientras estudia la pieza, mientras se estudia la performance [...]. A partir del momento en que subes al escenario y empiezas a pensar que el taxi que te tenía que llevar llegó veinte minutos tarde y te irritaste, y llegaste al teatro y discutiste con el iluminador porque estaban haciendo ruido durante el ensayo. En fin, todas esas cosas corrientes y mundanas, terminan interfiriendo en tu pensamiento, y se vuelven una especie de pensamiento intruso, muchas veces repetitivo, que te van dejando con un humor pésimo y una concentración muy baja. Entonces mi rutina consiste esencialmente en evitar ese tipo de cosas. (ZANON, 2018, información verbal) 
En relación a la actitud mental necesaria para enfrentar al público, varios entrevistados relataron estrategias de diálogo interno positivo (positive self-talk) y de búsqueda de motivación y conexión con el público. Fernández (2017, comunicación personal) destaca: “El público, incluso en el jurado de un concurso, no es hostil, está allí porque quiere oír. En una situación de concierto, lo que hacemos es tratar de compartir con ellos lo que hemos descubierto en nuestra preparación. Debe ser una fiesta y no un sufrimiento, para ninguna de las partes.”

En relación a la práctica instrumental, usualmente los entrevistados se concentran en tocar el repertorio completo durante los últimos días antes del concierto, estudiando principalmente la performance de las piezas y enfocándose en los puntos problemáticos: "usualmente toco el programa completo, repitiendo unas pocas cosas que me producen inseguridad” (MATARAZZO, 2017, comunicación personal); "hago un breve precalentamiento con escalas cromáticas y algunas de las secciones más difíciles a un tempo más lento” (BORG, 2017, comunicación personal). Durante el día del recital, fue frecuente en los relatos la preferencia por tocar el programa completo por lo menos una vez, con la salvedad de no colocar toda la energía que será necesaria después sobre el escenario, y sí haciéndolo de una forma tranquila y lenta.

Todos los momentos en que esté con el instrumento (que van a ser pocos, generalmente una hora antes de entrar al concierto), estaré tocando esos pasajes más complicados, pero de una forma lenta, bien tranquila y relajada. Todo eso también me va a ayudar para prepararme emocionalmente para entrar al concierto. (LOPES, 2017, comunicación personal)

Toco el repertorio una vez o dos veces y ya está [...]. Yo me levanto habitualmente el día del concierto y voy tocando el programa lentamente, parando donde hay problemas, pasando en la cabeza, pasando una obra, otra obra, la tercera obra, pausita... Porque para mí es importante pasarlo todo. Incluso si toco, no sé El Testamento de Amelia, que lo toco hace 30 años. (DUKIC, 2017, información verbal)

\subsection{Rutinas en la sala de conciertos}

Considerando los aspectos sobre la preparación en la sala de conciertos, los entrevistados comentaron la importancia de llegar con suficiente antecedencia (entre 45 minutos y una hora antes) al lugar del concierto para minimizar la ansiedad, probar la sala, practicar ejercicios de estiramiento y respiración, y poder realizar la preparación física y mental con calma. Con respecto a la prueba de 
sala, la mayoría de los guitarristas comentó que ocupa de 20 a 30 minutos en esa tarea, prestando atención a la comodidad de la silla y adaptándose a las luces y a la acústica del lugar. En relación al tiempo destinado al precalentamiento, los relatos fueron variados. Algunos de los concertistas comentaron dedicarle poco tiempo, y otros relataron utilizar aproximadamente media hora para realizar ejercicios de precalentamiento con el instrumento: "los últimos treinta minutos tengo un guión bien rígido, hago un precalentamiento con algunas piezas básicas y ejercicios para eso, y de acuerdo a la primera obra, toco una pieza o estudio para prepararme para la técnica básica que ella tenga” (MARCHIONE, 2017, comunicación personal); "me gusta calentar bastante antes de entrar al escenario. Toco durante media hora antes de entrar” (VIEAUX, 2017, comunicación personal).

Sobre tocar las obras del concierto antes de la performance, los relatos también fueron variados. Algunos, como en el caso de Matarazzo (2017) son determinantes en no tocar ninguna pieza del programa. Fernández (2017, comunicación personal), por ejemplo, repasa el programa completo en el camarín, "generalmente en el orden inverso, concentrando las fuerzas en la primera obra”; y Dukic (2017) habitualmente toca la mitad del programa en la prueba de sala. De la misma forma, fue comentado el hábito de tocar otras cosas antes de salir a escena — muchas veces improvisadas-, como por ejemplo "inventar una cantilena, vibrar cada nota [...] o tocar unas armonías bonitas" (IVANOVIĆ, 2017, información verbal), y enfocar toda la atención en la primera obra durante los últimos minutos antes de subir al escenario.

Varios relatos también hicieron referencia a la realización de ejercicios de respiración para lidiar con la ansiedad escénica y favorecer la concentración: "Lo único que hago bastante es Pranayama, ejercicios de respiración para bajar el nivel de estrés antes de entrar al escenario. Descubrí eso hace algunos años cuando competía, y es lo único que funciona para mí. Nunca tomé beta bloqueadores o cosas por el estilo.” (VILOTEAU, 2017, comunicación personal)

\section{Consideraciones finales}

A partir del análisis de estas entrevistas podemos destacar los aspectos más relevantes que los guitarristas de excelencia, que participaron de este estudio, consideran en la preparación para la performance en vivo. A pesar de las características individuales y fuertemente personales de cada uno 
(y de algunas diferencias de puntos de vista, como mencionamos a lo largo del análisis), se observaron aspectos en común en la manera en que piensan y estructuran la preparación para el escenario. De este modo, podemos concluir destacando la importancia de los siguientes factores:

(a) La creación de una imagen artística de la obra resulta fundamental en el inicio del proceso para identificar las dificultades técnicas, tomar decisiones expresivas e interpretativas y elegir digitaciones. Esto permite una optimización del tiempo de práctica, evitando la necesidad de tener que "reaprender" la obra al constatar que algunas ideas no funcionan. Este concepto se presenta como uno de los más relevantes en la preparación de una obra para la performance en vivo, corroborando los conceptos presentados por varios autores en las investigaciones empíricas (CHAFFIN, IMREH, 1997, 2002; HALLAM, 2001; CHAFFIN et al., 2003, 2010; CHAFFIN; LOGAN, 2006; CHAFFIN, 2007; LISBOA et al., 2018) y en la bibliografía guitarrística (RYAN, 1991; MASTERS, 2010).

(b) Los aspectos técnicos de la obra deben estar resueltos para considerar que está lista para tocar en público. De todas formas, este aspecto es solo la base y debe ser una herramienta que posibilite el desarrollo de los aspectos interpretativos, expresivos y técnicos.

(c) Las estrategias principales para el desarrollo de estos tres aspectos son: (1) análisis de la obra; (2) utilización de grabaciones como referencia; (3) grabación de la propia ejecución; (4) exploración de diferentes herramientas expresivas a partir de la realización de diferentes digitaciones, articulaciones, dinámicas, tempos, timbres, y aspectos gestuales; (5) la visualización con y sin partitura.

(d) Una vez que el aprendizaje de la obra está alcanzado (en otras palabras, se es capaz de realizar todos los movimientos necesarios para transmitir la idea interpretativa), es necesaria la práctica de la performance para trabajar sobre los aspectos propios que caracterizan la ejecución musical en vivo y para estar preparado para los posibles problemas que puedan surgir sobre el escenario. Esta práctica puede realizarse a través de simulaciones de la performance, de la grabación de la propia ejecución y de recitales informales.

(e) La digitación adquiere un rol fundamental en la práctica guitarrística. Esta puede ser pensada dependiendo de la finalidad (para una grabación o para tocar en vivo), y es importante probarla en situaciones de presión, ya que una digitación que no presenta problemas durante el estudio, puede no funcionar sobre el escenario. 
(f) La visualización constituye una herramienta fundamental y puede ser utilizada para diversos objetivos. A saber: (1) para aprender repertorio nuevo, (2) para resolver problemas técnicointerpretativos, (3) para crear la imagen artística de la obra, (4) para memorizar, (5) para lidiar con ansiedad escénica, (6) para favorecer la concentración, y (7) para prepararse para el escenario, imaginando la performance en vivo.

(g) A pesar de que algunos de los entrevistados consideren que la memorización no es relevante para tocar en vivo, para la mayoría, una performance memorizada favorece la expresividad, el desarrollo del lenguaje corporal, la seguridad en la ejecución, la comunicación, la espontaneidad y la posibilidad de tener todos los sentidos enfocados en la música.

(h) En los últimos días antes de tocar en público, el trabajo sobre la obra debe privilegiar el total por encima de las particularidades específicas, principalmente a partir de la realización de la práctica de la performance.

(i) En el día del recital es importante mantener una rutina tranquila, sin mucho esfuerzo físico y mental, manteniendo el foco en la performance evitando pensamientos negativos y reforzando los positivos, y alimentándose de una forma liviana para no tener problemas digestivos durante la ejecución. Con respecto al trabajo con el instrumento, es preferible no practicar demasiado, y solo realizar un pasaje del programa completo (de preferencia por la mañana), y dedicar un tiempo a los pasajes más problemáticos.

(j) A pesar de la imposibilidad de controlar todos los factores externos (público, acústica, otras situaciones imprevisibles) durante la ejecución en vivo, la práctica de la performance brinda herramientas para poder lidiar más eficazmente con los problemas mencionados.

Todos los aspectos analizados en las entrevistas con guitarristas de excelencia, constituyen una amplia fuente de posibilidades para el estudio e investigación de la aplicación práctica de estos conceptos. Las implicaciones didácticas de estas ideas, también constituyen un posible futuro campo de investigación, a través de la transformación de estos resultados en estrategias pedagógicas efectivas para la preparación de una performance en vivo de excelencia por parte de estudiantes de instrumento de diferentes niveles. 


\section{AGRADECIMIENTOS}

Agradecemos a la CAPES, por el financiamiento de esta investigación, a través de la beca de doctorado y doctorado sándwich del primer autor de este trabajo, y a la profesora Dra. Helena Marinho, por sus valiosos consejos, sugerencias y correcciones en la primera etapa de esta investigación. También queremos agradecer muy especialmente a todos los guitarristas participantes de esta investigación que cedieron parte de su valioso tiempo para compartir sus conocimientos y experiencias.

\section{REFERENCIAS}

ARTZT, Alice. Cuestionario aplicado por entrevista. Recibido por $<$ rafaeliravedra@hotmail.com $>$. 10 de abril de 2017.

ASSAD, Sérgio. Cuestionario aplicado por entrevista. Recibido por < rafaeliravedra@hotmail.com>. 1 de septiembre de 2018.

AZABAGIC, Denis. On Competitions: Dealing with Performance Stress. Pacific, MO: Mel Bay Publications, Inc., 2003.

Cuestionario aplicado por entrevista. Recibido por

$<$ rafaeliravedra@hotmail.com>. 11 de septiembre de 2018.

BANGERT, D. et al. Performing Solo Bach: A Case Study of Musical Decision-Making. Musicae Scientiae, v. 18, n. 1, p. 35-52, 2019.

BARROS, Luís Cláudio. A pesquisa empirica sobre o planejamento da execução instrumental: uma reflexão crítica do sujeito em um estudo de caso. 2018. Tesis (Doctorado en Música, Prácticas Interpretativas). UFRGS, Porto Alegre, 2008.

BORG, Scott. Cuestionario aplicado por entrevista. Recibido por <rafaeliravedra@hotmail.com>. 18 de abril de 2017.

BUNGARTEN, Frank. Cuestionario aplicado por entrevista. Recibido por

$<$ rafaeliravedra@hotmail.com>. 23 de abril de 2017.

CHAFFIN, Roger. Learning Clair de Lune: retrieval practice and expert memorization. Music Perception, v. 24, n. 4, p. 377-393, 2007.

CHAFFIN, Roger; IMREH, Gabriela. "Pulling teeth and torture": musical memory and problem solving. Thinking $\Xi^{2}$ Reasoning, v. 3, n. 4, p. 315-336, 1997.

. Practicing Perfection: Piano Performance as Expert Memory. Psychological

Science, v. 13, n. 4, p. 342-349, 2002.

CHAFFIN, Roger; LOGAN, Gabriela. Practicing perfection: How concert soloists prepare for performance. Advances in cognitive psychology, v. 2, n. 2-3, p. 113-130, 2006. 
CHAFFIN, R. et al. "Seeing the big picture": Piano Practice as Expert Problem Solving. Music Perception, v. 20, n. 4, p. 465-490, 2003.

CHAFFIN, R. et al. Preparing for memorized cello performance: the role of performance cues. Psychology of Music, v. 38, n. 1, p. 3-30, 2010.

CLARK, Terry; LISBOA, Tânia; WILLIAMON, Aaron. An investigation into musicians thoughts and perceptions during performance. Research Studies in Music Education, v. 36, n. 1, p. 19-37, 2014.

CLARKE, E. et al. Interpretation and Performance in Bryn Harrison's être-temps. Musicae Scientiae, v. 19, n. 1, p. 31-74, 2005.

DABENIGNO, Valeria. La sistematización de datos cualitativos desde una perspectiva procesual: De la transcripción y los memos a las rondas de codificación y procesamiento de entrevistas. In: BORDA, Pablo et al. Cuaderno 2: Estrategias para el Análisis de Datos Cualitativos. Buenos Aires: Serie Herramientas Para La Investigación Social, IIGG-UBA, 2017, p. 22-71.

DEMOS, A. P. et al. A longitudinal study of the development of expressive timing. Psychology of Music, p. 1-17, 2018.

DOĞANTAN-DACK, Mine. The art of research in live music performance. Music Performance Research, v. 5, p. 34-48, 2012.

DUKIC, Zoran. Entrevista concedida a Rafael Iravedra. Aveiro, Portugal, 30 de octubre de 2017. ESCARPA, Margarita. Entrevista concedida a Rafael Iravedra. Vigo, España, 8 de noviembre de 2017.

ESCOBAR, José Antonio. Entrevista concedida a Rafael Iravedra. Barcelona, España, 28 de noviembre de 2017.

FERNÁNDEZ, Eduardo. Técnica, Mecanismo, Aprendizaje. Una investigación sobre llegar a ser guitarrista. Montevideo: Ediciones ART, 2001

. Cuestionario aplicado por entrevista. Recibido por

$<$ rafaeliravedra@hotmail.com>. 10 de abril de 2017.

FREITAS, Stefanie; GERLING, Cristina Capparelli. Modelagem como estratégia de estudo para a manipulação das inflexões rítmicas e definição de íntimo no Ponteio 46 de Camargo Guarnieri: dois estudos de caso. Revista da ABEM, Londrina, v. 24, n. 36, p. 85-104, ene-jun 2016.

GARCÍA, Rafael. Entrenamiento mental para músicos. Técnicas de estudio mental y visualización para potenciar el rendimiento interpretativo. Redbook ediciones, Barcelona, 2017.

GARROBÉ, Àlex. Entrevista concedida a Rafael Iravedra. Barcelona, España, 28 de noviembre de 2017.

GINSBORG, Jane; CHAFFIN, Roger. Preparation and spontaneity in performance: A singer's thoughts while singing Schoenberg. Psychomusicology: Music, Mind Ev Brain, v. 21, n. 1-2, 2011.

GINSBORG, Jane; CHAFFIN, Roger; DEMOS, Alexander P. Different roles for prepared and spontaneous thoughts: A practice-based study of musical performance from memory. Journal of Interdisciplinary Music Studies, v. 6, n. 2, p. 201-231, 2012. 
GLISE, Anthony. Classical Guitar Pedagogy: A Handbook for Teachers. Pacific, MO: Mel Bay Publications, Inc., 1997.

GRONDONA, Stefano. Cuestionario aplicado por entrevista. Recibido por $<$ rafaeliravedra@hotmail.com>. 12 de abril de 2017.

HALLAM, Susan. Professional Musicians' Approaches to the Learning and Interpretation of Music. Psychology of Music, v. 23, n. 2, p. 111-128, 1995 a.

. Professional Musicians' Orientations to Practice: Implications for Teaching. British Journal of Music Education, v. 12, n. 1, p. 3-19, 1995 b.

. The development of memorisation strategies in musicians: Implications for education. British Journal of Music Education, v. 14, n. 1, p. 87-97, 1997.

. The development of metacognition in musicians: Implications for education. British Journal of Music Education, v. 18, n. 1, p. 27-39, 2001.

HÉROUX, Isabelle. Understanding the Creative Process in the Shaping of an Interpretation by Expert Musicians: Two Case Studies. Musicae Scientiae, v. 20, n. 3, p. 304-324, 2016.

. Creative Processes in the Shaping of a Musical Interpretation: A Study of Nine

Professional Musicians. Frontiers in Psychology, 9:665, 2018.

ISBIN, Sharon. Classical Guitar Answer Book. San Anselmo, Calif. : Milwaukee, Wis.: String Letter Publishing, 1999.

IVANOVIĆ , Dejan. Entrevista concedida a Rafael Iravedra. Guimarães, Portugal, 28 de diciembre de 2017.

IZNAOLA, Ricardo. On Practicing: A Manual for Students of Guitar Performance. Pacific, MO: Mel Bay Publications, Inc., 2001.

JØRGENSEN, Harald. Strategies for Individual Practice. In: WILLIAMON, Aaron (ed.). Musical Excellence: Strategies and Techniques to Enhance Performance. Oxford: Oxford University Press, 2004. p. 85-103.

KÄPPEL, Hubert. The Bible of Classical Guitar Technique. Germany: AMA Verlag, 2016.

LEATHWOOD, Jonathan. Cuestionario aplicado por entrevista. Recibido por

$<$ rafaeliravedra@hotmail.com>. 2 de septiembre de 2017.

LEISNER, David. Playing with ease: a healthy approach to guitar technique. Oxford University Press, 2018.

LISBOA, T. et al. Mastery through Imitation: A Preliminary Study. Musicae Scientiae, v. 19, n. 1, p. 75-110, 2005.

LISBOA, Tânia; DEMOS, Alexander P.; CHAFFIN, Roger. Training Thought and Action for Virtuoso Performance. Musicae Scientiae, v. 22, n. 4, p. 519-538, 2018.

LOPES, Edson. Cuestionario aplicado por entrevista. Recibido en formato de audio por $<$ rafaeliravedra@hotmail.com>. 11 de abril de 2017.

MARCHIONE, Carlo. Cuestionario aplicado por entrevista. Recibido por 
$<$ rafaeliravedra@hotmail.com>. 29 de junio de 2017.

MASTERS, Martha. Reaching The Next Level: A Method for the Experienced Classical Guitarist. Spi edition. Pacific, MO: Mel Bay Publications, Inc., 2010.

MATARAZZO, Lucio. Cuestionario aplicado por entrevista. Recibido por $<$ rafaeliravedra@hotmail.com>.9 de mayo de 2017.

MEJÍA NAVARRETE, J. Problemas centrales del análisis de datos cualitativos. Revista Latinoamericana de Metodologia de la Investigación Social, v. 1, p. 47-60, 2011.

MILES, Mathew B.; HUBERMAN, A. Michael. Qualitative data analysis: an expanded sourcebook. 2. ed. Thousand Oaks: SAGE Publications, 1994.

MILES, Mathew B.; HUBERMAN, A. Michael; SALDAÑA, Johnny. Qualitative data analysis: A Methods Sourcebook. 3. ed. Thousand Oaks: SAGE Publications, 2014.

NEUHAUS, Heinrich. The Art of Piano Playing. New York: Praeger Publishers, 1973.

PAPAGEORGI, Ioulia. Developing and Maintaining Expertise in Musical Performance. In: PAPAGEORGI, Ioulia; WELCH, Graham (Org.). Advanced Musical Performance. Investigations in Higher Education Learning. Surrey: Ashgate Publishing, 2014. p. 303-318.

PARTINGTON, John T. Making Music. Canada: Carleton University Press, 1995.

PEREIRA COUTINHO, Clara. Metodologia da Investigação em Ciências Sociais e Humanas: Teoria e Prática. Coimbra: Edições Almedina, 2011.

PEDREIRA RODRÍGUEZ, Martín. Ergonomía de la guitarra: su técnica desde la perspectiva corporal. La Habana, Cuba: Ediciones Cúpulas/Instituto Superior de Arte, 2011.

PÉREZ, Carlos. Cuestionario aplicado por entrevista. Recibido por $<$ rafaeliravedra@hotmail.com>. 5 de mayo de 2017.

PROVOST, Richard. The Art and Technique of Practice. Music Sales, 1992.

RUQUOY, Danielle. Situação de entrevista e estratégia do entrevistador. In: ALBARELLO, Luc et al. Práticas e Métodos de Investigação em Ciências Sociais. Tradução: Luísa Baptista. Lisboa: Gradiva, 1997, p. 84-116.

RYAN, Lee. The Natural Classical Guitar: Principles of Effortless Playing. USA: Bold Strummer Ltd, 1991.

SABARIEGO-PUIG, M.; VILÀ-BAÑOS, R.; SANDÍN-ESTEBAN, M. P. El análisis cualitativo de datos con ATLAS.ti. REIRE, Revista d'Innovació i Recerca en Educació, v. 7 n. 2, p. 119-133, 2014.

SHEARER, Aaron. Learning the Classic Guitar: Part 3. Pacific, MO: Mel Bay Publications, Inc., 1991.

SHERROD, Ronald J. Guitar master class: technical exercises by famous guitarists and teachers. Melville, N.Y: Belwin Mills, 1980.

SIEWERS, María Isabel. Entrevista concedida a Rafael Iravedra. Pelotas, Brasil, 27 de enero de 2017. 
TAMAYO, Marco. Entrevista concedida a Rafael Iravedra. Salzburgo, Austria, 10 de marzo de 2018. TENNANT, Scott. Pumping Nylon: The Classical Guitarist’s Technique Handbook. 2a edición. Alfred Music, 1995.

VAN ZIJL, Anemone G.W.; SLOBODA, John. Performers' Experienced Emotions in the Construction of Expressive Musical Performance: An Exploratory Investigation. Psychology of Music, v. 39, n. 2, p. 196-219, 2011.

VIEAUX, Jason. Cuestionario aplicado por entrevista. Recibido por $<$ rafaeliravedra@hotmail.com>. 26 de mayo de 2017.

VILOTEAU, Thomas. Dans la boîte noire: technique(s) de la guitare classique. 2. ed., 2012. E-book (98 p.).

Cuestionario aplicado por entrevista. Recibido por

$<$ rafaeliravedra@hotmail.com>. 26 de abril de 2017.

VINEY, Liam; BLOM, Diana. Preparing Stylistically Challenging Contemporary Classical Repertoire for Performance: Interpreting Kumari. International Journal of Music Education, v. 33, n. 1, p. 66-79, 2015.

VOLIOTI, Georgia; WILLIAMON, Aaron. Recordings as learning and practising resources for performance: Exploring attitudes and behaviours of music students and professionals. Musicae Scientiae, v. 21, n. 4, p. 499-523, 2017.

WOODY, Robert H. The motivations of Exceptional Musicians. Music Educators Journal, v. 90, n. 3, p. 17-21, 2004.

YATES, Stanley. Cuestionario aplicado por entrevista. Recibido por $<$ rafaeliravedra@hotmail.com>. 12 de abril de 2017.

ZANON, Fabio. Entrevista concedida a Rafael Iravedra. Vía Skype, 9 de febrero de 2018.

\section{SOBRE LOS AUTORES}

Rafael Iravedra es Doctor y Magister en Música-Prácticas Interpretativas (Guitarra) por la UFRGS. Realizó sus estudios de posgrado como becario CAPES bajo la orientación artística y académica del Prof. Dr. Daniel Wolff. Es profesor de música (orientación Guitarra) por la UNLP y cursó especialización en Guitarra con el maestro Eduardo Isaac. Ha tocado como solista e integrante de conjuntos de cámara en diversas salas de Argentina, Brasil, Colombia, Estados Unidos, México, España, Francia, Portugal, Bélgica y Alemania. En 2017 realizó doctorado sándwich en la Universidad de Aveiro (Portugal), y actualmente es pos-doctorando en la UFRGS. ORCID: https://orcid.org/0000-0002-4048-4033. E-Mail: rafael.iravedra@ufrgs.br 
Daniel Wolff es Profesor Titular de la Universidade Federal do Rio Grande do Sul y ex-Profesor visitante de la Universität der Künste Berlin (pos-doctorado). Obtuvo los títulos de Licenciado en Música (Universidad de la República, Montevideo, 1989), Magíster en Música (Manhattan School of Music, 1991, becado por la CAPES) y Doctor en Música (Manhattan School of Music, 1998, becado por el CNPq). Publicó artículos, libros y partituras en Brasil, Uruguay, Estados Unidos, Inglaterra, Alemania y Portugal. Lanzó 11 discos en Brasil, Uruguay y Alemania, con grabaciones solo, música de cámara y conciertos para guitarra y orquesta. ORCID: https://orcid.org/0000-0003-0839-909. E-Mail: daniel@danielwolff.com 